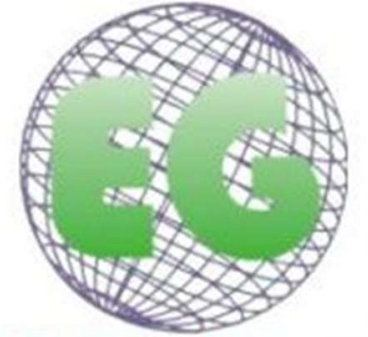

ISSN 1695-6141 $N^{\circ} 59$

\title{
Síndrome de burnout en el personal de enfermería de unidades de cuidado crítico y de hospitalización
}

Burnout syndrome in nursing personnel working in the critical care and hospitalization units

Militza Saraí Rendón Montoya ${ }^{1}$

Sandra Lidia Peralta Peña ${ }^{2}$

Eva Angelina Hernández Villa ${ }^{3}$

Reyna Isabel Hernández ${ }^{3}$

María Rubi Vargas ${ }^{2}$

María Alejandra Favela Ocaño 3

1 Estudiante de Especialidad de Enfermería en Cuidados Intensivos, Enfermera Especialista del Instituto Mexicano del Seguro Social. México.

2 Doctora en Enfermería, Docente de tiempo completo del Departamento de Enfermería de la Universidad de Sonora, México.

3 Maestra en Enfermería, Docente de tiempo completo del Departamento de Enfermería de la Universidad de Sonora, México. eva.hernandez@unison.mx

\section{https://doi.org/10.6018/eglobal.398221}

Recibido: $4 / 10 / 2019$

Aceptado: 9/11/2019

\section{RESUMEN:}

Introducción: El objetivo del estudio fue identificar la prevalencia del Síndrome de Burnout y sus dimensiones en el personal de enfermería de unidades de cuidado crítico y de hospitalización.

Metodología: Se llevó a cabo un estudio observacional descriptivo, transversal en 90 enfermeras y enfermeros. Se utilizó el Cuestionario de Maslach Burnout Inventory para el personal de salud. Se realizó análisis exploratorio y análisis descriptivo e inferencial; se emplearon los estadísticos $U$ de Mann Whitney y Kruskal Wallis.

Resultados: Se encontró nivel medio de Síndrome de Burnout 82.2\%; Agotamiento Emocional bajo 62.2\%; nivel bajo de Despersonalización $57.8 \%$ y nivel bajo de Falta de Realización Personal $40 \%$. Se evidenció diferencia estadísticamente significativa entre Síndrome de Burnout y turno de trabajo, doble turno al mes, períodos vacacionales al año y carga de trabajo; entre Agotamiento Emocional y tipo de servicio, ingreso quincenal, doble turno al mes, períodos vacacionales, tipo de contratación y carga de trabajo; entre Despersonalización y carga de trabajo; y entre Falta de Realización Personal y tipo de servicio, gusto por el servicio, turno de trabajo, ingreso quincenal, períodos de descanso en el turno, períodos vacacionales al año y tipo de contratación.

Conclusiones: Un porcentaje mayor del personal de enfermería obtuvo nivel medio de Síndrome de Burnout. No se encontró diferencia estadísticamente significativa entre el síndrome de Burnout y sus dimensiones con las características sociológicas del personal de enfermería. Se encontró evidencia de que las características laborales son las que presentan mayor influencia en el desarrollo del Síndrome de Burnout. 
Palabras clave: Síndrome de Burnout, Agotamiento Emocional, Despersonalización, Falta de Realización Personal, Personal de Enfermería.

\begin{abstract}
:
Introduction: The objective of the study was to identify the prevalence of the Burnout Syndrome and its dimensions in the nursing personnel working in the critical care and hospitalization units.

Methodology: A descriptive, observational, and cross-sectional study was performed in 90 nurses. The Maslach Burnout Inventory for health personnel was used. An exploratory, descriptive, and inferential analysis was carried out; the Mann Whitney $U$ and Kruskal Wallis tests statistics were used.

Results: A medium level regarding the burnout syndrome, $82.2 \%$, was found; emotional exhaustion decreased by $62.2 \%$; low level of depersonalization, $57.8 \%$, and low level of lack of personal accomplishment, $40 \%$. There was a statistically significant difference between burnout syndrome and work shift, double work shift per month, vacation periods per year, and workload; between emotional exhaustion and type of service, two-week income, double work shift, vacation periods, type of procurement, and workload; between depersonalization and workload; and between lack of personal accomplishment and type of service, work shift, two-week income, rest period during the shift, vacation periods per year, and type of procurement.

Conclusions: A higher percentage of nursing personnel had a mild burnout syndrome. No statistically significant difference between burnout syndrome and its dimensions and the sociological characteristics of the nursing personnel was found. Evidence that job characteristics are those that show more influence in the development of the burnout syndrome was found.
\end{abstract}

Key words: Burnout syndrome, emotional exhaustion, depersonalization, lack of personal accomplishment, nursing personnel.

\title{
INTRODUCCIÓN
}

Esta investigación tiene como propósito principal determinar la prevalencia de Síndrome de Burnout en el Personal de Enfermería que labora en las unidades de cuidado crítico y de hospitalización de un hospital de segundo nivel de atención. Los resultados de este estudio permitirán identificar los factores sociales y laborales del profesional de enfermería que influyen en el desarrollo del citado Síndrome.

El síndrome de Burnout (SB) ha sido definido como una respuesta que presenta la persona al estrés laboral crónico manifestado por actitudes y sentimientos negativos hacia los individuos con los que se trabaja y hacia el propio rol profesional, es la sensación de encontrarse emocionalmente agotado; esta respuesta ocurre más frecuentemente en los profesionales de la salud. El término Burnout, traducido como "estar quemado" fue introducido por Freudenberger a mediados de los años setenta para dar una explicación al proceso negativo que sufre el personal en su desempeño laboral. Se manifiesta como un deterioro en la atención personal a los pacientes de las instituciones que fundamentalmente prestan servicios ${ }^{(1,2)}$.

Posteriormente, en 1982, Maslach desarrolló un cuestionario basado en las respuestas de los trabajadores ante diferentes situaciones en su vida laboral cotidiana, para medir y valorar las 3 dimensiones básicas del síndrome: el agotamiento emocional (AE), la despersonalización (DP) y la falta de realización personal (FRP); es decir, se trata de un conjunto de conductas (disminución del rendimiento físico e intelectual, pérdida de responsabilidad, actitudes pasivo-agresivas con los receptores del servicio profesional, disminución de la motivación), en los que se involucran factores internos (valores individuales y sociales, rasgos de personalidad) como externos (organizacionales, laborales y grupales) ${ }^{(3)}$. 
Los profesionales de enfermería han sido identificados como uno de los principales grupos de riesgo para el desarrollo del SB o síndrome del "quemado". Desde una perspectiva psicosocial, varios autores admiten la definición propuesta por Maslach y Jackson, quienes señalan que el SB deber asumirse como un síndrome de agotamiento emocional, despersonalización y falta de realización personal en el contexto laboral, que se desarrolla como resultado de la exposición permanente a distintos estresores en el trabajo ${ }^{(4)}$.

Los sujetos afectados por este síndrome muestran desilusión, irritabilidad, enojo, sentimientos de frustración, actitudes suspicaces, rigidez, inflexibilidad y obstinación. Así mismo, como consecuencia afloran síntomas de carácter psicosomático y se produce deterioro de la salud en las personas afectadas por este síndrome. Los afectados se identifican con sintomatología que se integra en cuatro grandes grupos en función de sus manifestaciones: emocional, actitud, conductual y psicosomático ${ }^{(3}$, $5)$.

Los síntomas cardinales del SB son: sensación de realización personal baja, tendencia a autoevaluación negativa, altos niveles de agotamiento emocional, expresión verbal de incapacidad para dar más de sí en la solución de la problemática del receptor del cuidado, trato deshumanizado o despersonalización, desarrollo de comportamientos que no corresponden al trato habitual de la persona y cinismo debido a una coraza emocional, entre otros. Las manifestaciones de acuerdo con la gravedad del síndrome, se clasifican en: leves (quejas inespecíficas y frecuentes, cansancio, pocas ganas de acudir al trabajo, dificultad para levantarse en la mañana); moderadas (cinismo, aislamiento, suspicacia y negativismo); graves (ausentismo laboral, desempeño lento de las actividades, automedicación, aversión y abuso de drogas lícitas e ilícitas); y finalmente, extremas (aislamiento social marcado, colapso, cuadros psiquiátricos y suicidios) ${ }^{(6)}$.

El contexto laboral de los profesionales de enfermería está definido por problemas relacionados con condiciones laborales marcadas por la participación de riesgos psicosociales, jornadas extenuantes y extensas, sobrecarga de trabajo, turnos rotatorios, cambios de servicio no programados y estrés por presencia de situaciones críticas y demandantes con los usuarios, familiares e incluso con colegas y profesionales del equipo de trabajo. Estos hechos pueden conducir a una tendencia progresiva en el desarrollo de riesgos ocupacionales que a su vez pueden originar alteraciones fisiológicas ${ }^{(6-8)}$.

Estudios internacionales ponen en evidencia que un menor número de enfermeras(os) se asocia a mayor mortalidad y morbilidad de los pacientes y a una peor calidad en el cuidado profesional ${ }^{(9,10)}$. Asimismo, se han mostrado evidencias de que el personal de enfermería aumenta la probabilidad de ausentarse de su jornada laboral debido a enfermedades donde las principales causas son el estrés y la falta de satisfacción en el trabajo ${ }^{(11)}$.

De esta manera, la jornada de trabajo se convierte en un elemento que favorece el desgaste y sufrimiento en los trabajadores; cuando el ambiente organizacional induce sufrimiento, el individuo desarrolla mecanismos de defensa para enfrentarlo y manejarlo sin que afecte la humanidad de la propia persona. Sin embargo, cuando se incrementan los problemas y el trabajador entra en agotamiento emocional, frustración y el trabajo no le permite realizarse, los ambientes de las organizaciones pueden volverse más estresantes, incluso amenazantes para los trabajadores ${ }^{(8)}$. 
Hoy en día el SB, constituye un factor de riesgo laboral frecuente en el personal de enfermería. Es una enfermedad que ha sido reconocida por varios estudiosos de psicología y sociología laboral. Sus manifestaciones se presentan gradualmente en el personal de enfermería y si no se identifican y tratan con oportunidad progresan y se agravan, de manera que pueden causar incapacidad y ausentismo laboral, e incluso la muerte laboral y social del trabajador, a causa de excesivo agotamiento emocional(12).

Este síndrome, que significa "quemarse en el trabajo", ha sido analizado por expertos nacionales e internacionales de enfermería; ellos señalan la gravedad del problema que vive este grupo de profesionales al enfrentarse cotidiana y permanentemente al estrés en sus actividades, en instituciones de salud, tanto públicas como privadas. La necesidad de estudiarlo como un factor de riesgo que conduce a patología laboral, se une con los requerimientos de analizar los procesos de estrés laboral, así como el interés que actualmente tienen algunas organizaciones por sus empleados, para otorgarles mejor calidad de vida laboral ${ }^{(13) .}$

Por ello, y debido a que la enfermería es una de las profesiones que está sometida a altos niveles de estrés y que éste impacta en la calidad de los servicios que otorga, se hace necesario evaluar su actuación para mantener y promover un clima laboral satisfactorio, con importantes resultados no solo para la excelencia de los cuidados de los pacientes, si no especialmente para el personal de enfermería como trabajador de salud que es el que promueve el cuidado.

\section{MATERIAL Y MÉTODOS}

Se realizó un estudio observacional, descriptivo con un diseño transversal. La población de estudio estuvo constituida por el personal de enfermería que labora en un hospital público de segundo nivel de atención del Estado de Sonora, México. La muestra fue probabilística, se estimó por medio del programa STATS, con un nivel de confianza de 0.95 y margen de error de 0.05. El tamaño de la muestra fue de 90 enfermeras y enfermeros que prestaban cuidado directo a pacientes laborando en los servicios hospitalarios seleccionados. Para la recolección de datos de este estudio se utilizó un cuestionario integrado por 39 preguntas, 22 de ellas corresponden al Maslach Burnout Inventory (MBI) y 17 a variables sociológicas y laborales.

En las variables sociológicas se consideraron los indicadores de edad, género, estado civil, número de hijos y en las laborales se incluyó a los servicios hospitalarios, el gusto por el servicio, turno laboral, doble turno por mes, preparación profesional, horas de trabajo por semana, descansos en el turno, carga de trabajo, periodos de descanso, tipo de contratación, antigüedad laboral, sueldo quincenal y períodos vacacionales al año.

Respecto la medición de la variable dependiente se utilizó el Cuestionario de Maslach Burnout Inventory para el personal sanitario (MBI-HSS [Maslach Burnout Inventory Human Services Survey] por sus siglas en inglés): este cuestionario diseñado por Maslach y Jackson, permite valorar la presencia del Síndrome de Burnout en el personal sanitario y ha sido validado en población latinoamericana y en población mexicana ${ }^{(14,15)}$.

Este cuestionario está constituido por 22 ítems en forma de afirmaciones medidos en una escala de Likert de 0 (nunca) a 6 (todos los días) puntos, indaga sobre los 
sentimientos y actitudes del profesional en su trabajo y hacia los pacientes. La puntuación puede fluctuar de 0 a 132 puntos y el resultado es directamente proporcional a la presencia del SB. El MBI-HSS se autocomplementa en 10 a 15 minutos y mide las tres dimensiones del síndrome: agotamiento emocional, despersonalización y la falta realización personal. Se clasifica en tres niveles, bajo ( $\leq$ $43)$, medio (44-87) y alto ( $\geq 88)$.

Esta variable fue evaluada también a través de sus dimensiones y los valores asignados a las mismas, tal como se presentan en la tabla 1:

Tabla 1: Valores de las dimensiones del Maslach Burnout Inventory.

\begin{tabular}{|c|c|c|c|}
\hline & Bajo & Medio & Alto \\
\hline $\begin{array}{c}\text { Agotamiento } \\
\text { Emocional }\end{array}$ & $\leq 18$ & $19-26$ & $\geq 27$ \\
\hline $\begin{array}{c}\text { Despersonalización } \\
\begin{array}{c}\text { Falta de realización } \\
\text { personal }\end{array}\end{array}$ & $\leq 5$ & $6-9$ & $\leq 33$ \\
\hline
\end{tabular}

El procedimiento para la recolección de información, se llevó a cabo durante los meses de noviembre y diciembre del 2017 en los servicios de Cirugía, Ginecología, Medicina Interna, Pediatría, Unidad de Cuidados Intensivos (UCI), Unidad de Cuidados Intensivos Neonatales (UCIN), Unidad de Cuidados Intensivos Pediátricos (UCIP), Unidad de Terapia Intermedia (UTI) y Urgencias del hospital seleccionado. Previo al llenado del cuestionario se solicitó el consentimiento informado a través de la lectura y firma de un documento diseñado para ello ${ }^{(16)}$.

Los datos obtenidos fueron capturados en el programa estadístico SPSS, versión 22.0 (Statistical Package for the Social Sciences). Para caracterizar a los sujetos de estudio, se realizó análisis preliminar descriptivo (medidas de tendencia central y dispersión) para las variables continuas y discretas; se realizaron pruebas de Kolmogorov Smirnov, para evaluar la normalidad de los datos. Las variables dependientes no tuvieron distribución normal por lo que se empleó estadística no paramétrica. La diferencia de medianas y significancia estadística se evaluó con $U$ de Mann Whitney y Kruskal Wallis.

La investigación se llevó a cabo posterior a la obtención del dictamen favorable del Comité de Ética en Investigación del Departamento de Enfermería de la Universidad Sonora y de la institución de salud donde se realizó el estudio como lo dispone la normatividad relativa a la investigación en seres humanos ${ }^{(16)}$.

\section{RESULTADOS}

En relación a las variables sociodemográficas se encontró que la muestra fue conformada mayoritariamente por mujeres, con una media de edad de 33.7 años, casadas en un $46.7 \%$ y con uno a dos hijos. Un poco más de la mitad de las enfermeras (os) se ubicaron en servicios de hospitalización y el $56 \%$ manifestó gusto por el servicio en el que se encontraban, con mayor porcentaje en los turnos matutino y nocturno, con jornadas de 31 a 40 horas, con salario quincenal de 3001 a 5000 
pesos, casi la mitad de los participantes tienen de 1 a 5 años de antigüedad, representando solo el $11 \%$ quienes tienen más de 21 años laborando, lo que se relaciona con que el hecho de que la mayoría no es personal de base y trabaja de 1 a 2 turnos dobles por mes, casi un $25 \%$ no goza de vacaciones y el resto tiene de 1 a 3 períodos anuales. Entre otras características consideran que su carga de trabajo es moderada y $82.2 \%$ toma de tres a cuatro períodos de descanso durante la jornada; solo el $21 \%$ tiene otro trabajo y la mayor parte del personal posee el grado de licenciatura en enfermería.

En relación a los resultados obtenidos para el Síndrome de Burnout se observa que el puntaje exhibido tiende a ser medio, puntaje promedio de 56.57; para agotamiento emocional tiende a ser bajo con una media de 15.32, lo mismo para despersonalización con una media de 5.13 y resultando alto para falta de realización personal con una media de 36.1 (Tabla 2).

Tabla 2: Distribución del personal de enfermería según Síndrome de Burnout, sus dimensiones y nivel

\begin{tabular}{|c|c|c|c|}
\hline Síndrome de Burnout, dimensiones y nivel & $n$ & $\%$ & MTC* \\
\hline $\begin{array}{l}\text { Síndrome de Burnout }(0-132) \\
\text { Alto }(\geq 88) \\
\text { Medio }(44-87) \\
\text { Bajo }(\leq 43)\end{array}$ & $\begin{array}{r}3 \\
74 \\
13\end{array}$ & $\begin{array}{r}3.3 \\
82.2 \\
3.3\end{array}$ & $\begin{array}{c}\bar{X}=56.57 \\
D E=17.08\end{array}$ \\
\hline $\begin{array}{l}\text { Agotamiento emocional }(0-54) \\
\text { Alto }(\geq 27) \\
\text { Medio }(19-26) \\
\text { Bajo }(\leq 18)\end{array}$ & $\begin{array}{l}17 \\
17 \\
56\end{array}$ & $\begin{array}{l}18.9 \\
18.9 \\
62.2\end{array}$ & $\begin{array}{c}\bar{X}=15.32 \\
D E=10.10\end{array}$ \\
\hline $\begin{array}{l}\text { Despersonalización }(0-30) \\
\text { Alto }(\geq 10) \\
\text { Medio }(6-9) \\
\text { Bajo }(\leq 5)\end{array}$ & $\begin{array}{l}19 \\
19 \\
52\end{array}$ & $\begin{array}{l}21.1 \\
21.1 \\
57.8\end{array}$ & $\begin{array}{c}\bar{X}=5.13 \\
D E=5.26\end{array}$ \\
\hline $\begin{array}{l}\text { Falta de realización personal }(0-48) \\
\text { Alto }(\leq 33) \\
\text { Medio }(34-39) \\
\text { Bajo }(\geq 40) \\
\end{array}$ & $\begin{array}{l}26 \\
28 \\
36\end{array}$ & $\begin{array}{l}28.9 \\
31.1 \\
40.0\end{array}$ & $\begin{array}{c}\bar{X}=36.11 \\
D E=11.01\end{array}$ \\
\hline
\end{tabular}

*MTC, Medidas de tendencia central; $\bar{X}$, media; $D E$, desviación estándar.

Fuente: Instrumento

( ) Punto de corte para la clasificación de niveles en cada dimensión.

Respecto a la clasificación establecida y su distribución porcentual se encuentra que el $82.2 \%$ del personal de enfermería presenta nivel medio de SB, en AE el $62.2 \%$ del personal presenta nivel bajo y un porcentaje igual (18.8\%) presenta $A E$ alto y medio. La dimensión de DP indica nivel bajo en el $57.8 \%$ del personal, mientras que el nivel alto y medio, estuvo presente en un porcentaje igual en ambos niveles $(21.1 \%)$. Se encontró que el $40 \%$ tiene un bajo nivel de FRP, $31.1 \%$ presentó nivel medio y 28.9 un alto nivel de esta dimensión (Tabla 2).

Al estudiar las relaciones existentes entre el Síndrome de Burnout, y las características sociológicas del personal de enfermería se encontró que la presencia del SB y sus dimensiones, es independiente del grupo de edad, género, estado civil y número de hijos del personal de enfermería. 
Tabla 3: Cruce de dos variables con Significancia Estadística

\begin{tabular}{|c|c|c|c|c|}
\hline VARIABLES & $\begin{array}{c}\text { Síndrome de } \\
\text { burnout }\end{array}$ & $\begin{array}{l}\text { Agotamiento } \\
\text { emocional }\end{array}$ & Despersonalización & $\begin{array}{l}\text { Falta de } \\
\text { realización } \\
\text { personal }\end{array}$ \\
\hline $\begin{array}{l}\text { Tipo de } \\
\text { servicio }\end{array}$ & $\begin{array}{l}U=913.0, p= \\
.458\end{array}$ & $\begin{array}{l}U=686.5, p= \\
.010\end{array}$ & $U=793.0, p=.083$ & $\begin{array}{l}U=628.5, p= \\
.002\end{array}$ \\
\hline $\begin{array}{l}\text { Gusto por el } \\
\text { servicio }\end{array}$ & $\begin{array}{l}H=.523, p= \\
.770\end{array}$ & $\begin{array}{l}H=1.636, p= \\
.441\end{array}$ & $H=3.291, p=.193$ & $\begin{array}{l}H=12.086, p= \\
.002\end{array}$ \\
\hline $\begin{array}{l}\text { Turno de } \\
\text { trabajo }\end{array}$ & $\begin{array}{l}H=15.662, p \\
=001\end{array}$ & $\begin{array}{l}H=1.704, p= \\
.636\end{array}$ & $H=1.797, p=.616$ & $\begin{array}{l}H=20.568, p= \\
.000\end{array}$ \\
\hline $\begin{array}{l}\text { Ingreso } \\
\text { quincenal }\end{array}$ & $\begin{array}{l}H=.179, p= \\
.915\end{array}$ & $\begin{array}{l}H=7.563, p= \\
.023\end{array}$ & $H=.573, p=.751$ & $\begin{array}{l}H=9.828, p= \\
.007\end{array}$ \\
\hline $\begin{array}{l}\text { Antigüedad } \\
\text { laboral }\end{array}$ & $\begin{array}{l}H=.567, p= \\
989\end{array}$ & $\begin{array}{l}H=.313, p= \\
.997\end{array}$ & $H=1.743, p=.883$ & $\begin{array}{l}H=1.382, p= \\
.926\end{array}$ \\
\hline $\begin{array}{l}\text { Doble turno } \\
\text { al mes }\end{array}$ & $\begin{array}{l}H=7.390, p= \\
.007\end{array}$ & $\begin{array}{l}H=4.462, p= \\
.035\end{array}$ & $H=1.973, p=.160$ & $\begin{array}{l}H=.328, p= \\
.567\end{array}$ \\
\hline $\begin{array}{l}\text { Períodos de } \\
\text { descanso en } \\
\text { el turno }\end{array}$ & $\begin{array}{l}H=4.426, p= \\
.219\end{array}$ & $\begin{array}{l}H=.723, p= \\
.868\end{array}$ & $H=.337, p=.953$ & $\begin{array}{l}H=8.150, p= \\
.043\end{array}$ \\
\hline $\begin{array}{l}\text { Periodos } \\
\text { vacacionales } \\
\text { al año }\end{array}$ & $\begin{array}{l}H=7.983, p= \\
.046\end{array}$ & $\begin{array}{l}H=8.079, p= \\
.044\end{array}$ & $H=5.471, p=.140$ & $\begin{array}{l}H=15.676, p= \\
.001\end{array}$ \\
\hline $\begin{array}{l}\text { Tipo de } \\
\text { contratación }\end{array}$ & $\begin{array}{l}H=5.334, p= \\
.069\end{array}$ & $\begin{array}{l}H=10.570, p= \\
005\end{array}$ & $H=.384, p=.825$ & $\begin{array}{l}H=10.405, p= \\
.006\end{array}$ \\
\hline $\begin{array}{l}\text { Carga de } \\
\text { trabajo }\end{array}$ & $\begin{array}{l}H=13.348, p \\
=001\end{array}$ & $\begin{array}{l}H=8.732, p= \\
.013\end{array}$ & $H=6.541, p=.038$ & $\begin{array}{l}H=1.615, p= \\
.446\end{array}$ \\
\hline
\end{tabular}

Fuente: Instrumento

Abreviaturas: $U$, prueba de Mann Whitney; $H$, prueba de Kruskal Wallis; $p$, valor para diferencia de medianas.

Entre las características laborales se destaca que el SB y la DP no presentan diferencia significativa de acuerdo al servicio en que se labora, sin embargo, el AE y la FRP sí la mostraron, encontrando que el personal de enfermería que labora en unidades de hospitalización presenta más agotamiento emocional que el de unidades de cuidado crítico, por el contrario, el que labora en unidades de cuidado crítico, presenta menor falta de realización personal.

La FRP presentó asociación con el gusto por el servicio. Al personal de enfermería al que le gusta mucho el servicio donde labora, presenta menos falta de realización personal que aquel personal al que el servicio no le gusta o le gusta más o menos. El $A E$ y la DP no presentaron diferencia significativa en relación con el turno de trabajo en que labora el personal de enfermería. Sin embargo, se observó que los niveles de SB y la FRP si presentaron diferencia significativa según el turno de trabajo. El personal de enfermería que labora en turno matutino, vespertino y nocturno, presenta mayor puntuación en SB y menor FRP que los que laboran en jornada acumulada (Tabla 3) y la presencia del SB y sus dimensiones, es independiente del número de horas que labora a la semana el personal de enfermería.

Los niveles de SB y de DP no presentaron diferencia significativa según el ingreso quincenal del personal de enfermería, pero la FRP y de AE sí mostraron asociación entre ellas. El personal de enfermería que percibe de tres mil uno a cinco mil pesos presenta mayor $\mathrm{AE}$ que los otros grupos, así como el personal de enfermería que percibe más de cinco mil pesos quincenales, presenta menos FRP que aquel que 
percibe de dos mil a cinco mil pesos, (Tabla 3). Los niveles de SB y sus dimensiones no presentaron diferencia significativa de acuerdo a la antigüedad laboral; la presencia del SB y sus dimensiones, son independientes del número de años que ha laborado el personal de enfermería en la institución de salud.

Además, se observa que el $\mathrm{SB}$ y el $\mathrm{AE}$, sí presentaron diferencia significativa de acuerdo al número de veces que el personal dobla turno en un mes. El personal de enfermería que dobla de 1 a 2 veces y 5 o más veces, presenta mayor puntuación en SB que aquel que dobla turno 3 a 4 veces al mes y el personal que dobla turno de 1 a 2 veces al mes presenta más $A E$, (Tabla 3 ).

Se encontró que la FRP muestra diferencia significativa en relación con los descansos durante la jornada laboral. El personal de enfermería que no toma ningún descanso durante la jornada laboral presenta mayor FRP que aquel que toma algún descanso en la jornada, (Tabla 3). De la misma manera se evidenció que los niveles de SB, el $A E$ y la $F R P$ si presentaron diferencia significativa según el número de periodos vacacionales. El personal de enfermería que no toma ningún período vacacional presenta mayor puntuación en el SB y presenta más $A E$ que aquel que toma de 1 a 2 períodos, por el contrario, el que toma 3 períodos, presenta menor FRP, (Tabla 3).

En la relación al tipo de contratación que tiene el personal de enfermería con los niveles de AE y FRP sí presentaron diferencias significativas. El personal de enfermería contratado como suplente fijo, presenta más $A E$ que aquel contratado como eventual o de base; por el contrario, el que tiene contrato de base, presenta menor FRP, (Tabla 3).

El personal de enfermería que considera alta su carga de trabajo presenta mayor puntuación en el SB, en AE y DP que aquel que considera baja o moderada su carga de trabajo, (Tabla 3)

\section{DISCUSIÓN}

Los resultados encontrados, muestran que el personal de enfermería presenta problemática con el Síndrome de Burnout, se observó que el puntaje exhibido por los participantes en la escala de medición tiende a ser medio $(M d n=55.0)$ y que la mayoría del personal (82.2\%) presentó nivel medio de SB. Algunos estudios realizados en México (17-20) y otros realizados fuera de México (21-23) encontraron prevalencias inferiores de nivel medio de Burnout; en cambio, un estudio realizado en España y Perú ${ }^{(2,24)}$ y otro en México (1) reportaron prevalencias superiores a la encontrada en este estudio.

En relación a la dimensión de agotamiento emocional, los resultados muestran que el puntaje observado en la escala de medición tiende a ser bajo $(M d n=14.5)$ y que la mayoría del personal (62.2\%) presentó nivel bajo de $\mathrm{AE}$, algunos estudios realizados en México $(17,19,20)$ y otros realizados fuera de México $(2,17,19,20)$ encontraron prevalencias superiores de nivel bajo de $\mathrm{AE}$; sin embargo un estudio realizado en México ${ }^{(18)}$ y otros realizados fuera de México ${ }^{(6,22,25)}$, reportaron prevalencia similar a la encontrada en este estudio.

Sobre la dimensión de despersonalización, los resultados muestran que el puntaje observado en la escala de medición tiende a ser muy bajo $(M d n=3.0)$ y que la 
mayoría del personal $(57.8 \%)$ presentó nivel bajo de DP. Un estudio mostró un valor de mediana similar (20). Además, algunos estudios realizados en México ${ }^{(17-20)}$ y otros realizados fuera de México ${ }^{22,26)}$, encontraron prevalencias superiores de nivel bajo de DP; sin embargo, un estudio realizado en España reportó prevalencia similar a la encontrada en este estudio ${ }^{(25)}$.

En relación a la dimensión de FRP, los resultados muestran que el puntaje observado en la escala de medición tiende a ser alto $(M d n=38.0)$ y que la mayoría del personal $(40.0 \%)$ presentó nivel bajo de FRP. Un estudio realizado en Perú ${ }^{(24)}$, mostró un valor de mediana similar $(M d n=42.0)$. Además, algunos estudios realizados en México $(17,19,20)$ y otros realizados fuera de México $(22,25,26)$, encontraron prevalencias inferiores de nivel bajo de FRP; se encontró además un estudio realizado en Colombia ${ }^{(21)}$ y otro en España ${ }^{(6)}$ que de prevalencia similar a la encontrada en este estudio.

Los resultados del estudio indicaron que no existe diferencia significativa entre el SB y sus dimensiones con la variable género. Estos resultados no concuerdan con algunas investigaciones que reportaron que el género femenino tiene mayor riesgo de desarrollar Burnout. $(2,18,19,23,27)$ Contrariamente otros estudio apoya que el personal masculino es quien presenta SB ${ }^{(21,22)}$.

En cuanto a la edad no se obtuvo diferencia significativa con ninguna de las dimensiones del SB. Los estudios revisados establecen resultados diferentes: se presenta en mayores de 50 años ${ }^{(27)}$ y entre los 35 a 44 años. ${ }^{(23)}$ o menores de 45 años (2). Un estudio realizado en Colombia ${ }^{(26)}$ que coincide, no reportó diferencia significativa entre el desarrollo del SB y la edad.

Respecto a la variable estado civil no se obtuvo relación significativa con el SB y sus dimensiones; estos resultados son compatibles con un estudio realizado en Perú (24), pero diferente a lo reportado en Colombia ${ }^{(21)}$ donde se encontró que el SB tuvo mayor prevalencia entre quienes no tenían una relación de pareja estable. La presencia de Burnout y sus dimensiones no presentó diferencia significativa según el número de hijos. En oposición, un estudio realizado en Colombia ${ }^{(21)}$, encontró que el riesgo de presentar SB se incrementa 2.5 veces en el personal que tiene hijos con respecto al que no tiene hijos. De igual manera Miranda Lara, en México reportó diferencia significativa entre SB y tener 2 y 3 hijos ${ }^{(27)}$.

Es interesante resaltar que ninguna de las variables sociológicas estudiadas en esta investigación se relacionó con el SB y sus dimensiones, este resultado es consistente con el encontrado en Colombia ${ }^{(26)}$ ya que al igual que este estudio, no se observó diferencia significativa con ninguna de las variables sociológicas estudiadas.

En el análisis de la variable servicio, contrariamente a lo esperado, el personal que labora en unidades de cuidado crítico presenta menor agotamiento emocional y menor falta de realización personal que el que labora en unidades de hospitalización. Una investigación realizada en España (25) obtuvo resultados similares, el personal que labora en unidades medico quirúrgicas presentó mayor prevalencia de $\mathrm{SB}$, de $\mathrm{AE}$ y DP que el que labora en unidades de cuidados críticos, sin embargo, ese mismo estudio reportó que quien labora en unidades de cuidado crítico, presenta mayor realización personal que los que trabajan en unidades medico quirúrgicas, mientras otro estudio identificó mayor prevalencia de SB en el área de psiquiatría ${ }^{(6)}$. También se reportaron resultados similares en México ${ }^{(27)}$ donde encontraron que el SB se presenta significativamente más en quien no labora en áreas críticas. 
El personal de enfermería que labora en los turnos matutino, vespertino y nocturno, presenta mayor puntuación de SB y menor FRP que los que laboran en jornada acumulada. Un estudio realizado en Colombia ${ }^{(26)}$ encontró diferencia significativa, el personal que labora en el turno vespertino, presenta mayor AE que el de los otros turnos. Para algunos autores ${ }^{(12,28)}$ el trabajo en el turno nocturno o la rotación constante entre turnos facilita la presencia del SB.

En la antigüedad laboral se muestra que no se encontró diferencia significativa con el SB y sus dimensiones; estos resultados son congruentes con los reportados por Arias (24) en Perú. En cambio, en España ${ }^{(2,23)}$ se reportó que el SB se presenta en los primeros años (1 a 8) de vida laboral; en Perú (22) también se encontró que los trabajadores con menos tiempo de antigüedad presentan niveles altos de DP.

El personal de enfermería contratado como suplente fijo, presenta mayor puntuación en el SB y presenta más AE que aquel contratado como eventual o de base; por el contrario, el que tiene contrato de base, presenta mayor FRP. Los resultados son consistentes con los encontrados en España ${ }^{(23)}$ y Colombia ${ }^{(26)}$ donde los resultados mostraron que el personal contratado como eventual presenta más AE y en Perú (22) donde el personal eventual presenta más FRP que los de contrato fijo.

Con respecto a la carga de trabajo los resultados describen diferencia significativa dado que el personal de enfermería que presenta mayor puntuación en el SB, presenta más $A E$ y mayor $D P$ es aquel que considera alta o moderada su carga de trabajo. El SB, el AE, la DP y la de FRP no presentaron diferencia significativa según la preparación profesional del personal de enfermería; sin embargo, se debe resaltar que los licenciados en enfermería presentaron prevalencias más elevadas que el resto de las categorías, especialmente en el nivel medio del SB. Un estudio realizado en Colombia (21) reveló resultados muy similares, en cambio, en España (3) se encontró que la falta de formación profesional está relacionada con mayor cansancio emocional y menor realización personal.

En los resultados se observa que los niveles de SB y sus dimensiones no presentaron diferencia significativa según el número de trabajos que tiene el personal de enfermería; Estos resultados son similares a los reportados por Miranda Lara en México ${ }^{(28)}$.

En general, las dimensiones en las que se encontró más características laborales con diferencia significativa fueron $A E$ y FRP, mientras que en DP solo se reportó una variable laboral con diferencia significativa.

Algunos de los resultados exhibidos no son compatibles con otras investigaciones, quizá por las características de los participantes, las variables que se emplearon, las condiciones del momento temporal de la aplicación, el ámbito y la cultura de la población en la que se desarrollaron los estudios y las condiciones laborales de las instituciones de salud en México.

Es importante destacar cómo algunos factores de la organización laboral en la que trabaja el personal de enfermería pueden intervenir en el nivel de SB y de sus tres dimensiones. Considerando el extenso número de variables que median en el referido síndrome y las implicaciones que tiene en la calidad del cuidado a la salud de los usuarios y del mismo personal de enfermería, es posible influir en las mismas, realizando las modificaciones adecuadas y oportunas en la organización y estructura de los servicios de enfermería. 
Una de las limitaciones del estudio es que algunas relaciones estudiadas no fueron significativas estadísticamente, sobre todo aquellas asociadas con las características sociológicas de la población de estudio. Esta situación puede deberse a que el tamaño de la muestra fue pequeño $(n=90)$ aunque, la tasa de respuesta obtenida fue alta.

\section{CONCLUSIONES}

En el estudio participaron 90 enfermeras y enfermeros de un hospital público de segundo nivel de atención; la mayoría mostró nivel medio de $S B$, nivel bajo de $A E$; nivel bajo de DP; y nivel bajo de FRP. No se encontró relación entre SB y sus dimensiones con las características sociales del personal de enfermería.

Se evidenció relación entre SB y las siguientes características laborales: turno de trabajo, realización de doble turno al mes, periodos vacacionales al año y carga de trabajo.

No se encontró relación entre SB y las características laborales que a continuación se mencionan: tipo de servicio, gusto por el servicio, horas de trabajo por semana, ingreso quincenal, antigüedad laboral, períodos de descanso en el turno, tipo de contratación, preparación profesional, y número de trabajos.

Se estableció asociación entre AE y las siguientes características laborales: tipo de servicio, ingreso quincenal, realización de doble turno al mes, periodos vacacionales al año, tipo de contratación y carga de trabajo.

Se mostró relación entre DP y la carga de trabajo.

Se encontró relación entre FRP y las siguientes características laborales: tipo de servicio, gusto por el servicio, turno de trabajo, ingreso quincenal, períodos de descanso en el turno, periodos vacacionales al año, y tipo de contratación.

La evidencia resultante del presente estudio sugiere que las características laborales son las que tienen mayor influencia en el desarrollo del SB y sus dimensiones en el personal de enfermería.

\section{REFERENCIAS}

1. Rodríguez García C, Oviedo AM, Vargas Santillán MdL, Hernández Velázquez V, Pérez Fiesco MdS. Prevalencia del Burnout Syndrome en el personal de Enfermería de dos Hospitales del Estado de México. Fundamentos en humanidades. 2009(19):179-93. http://www.redalyc.org/pdf/184/18411965011.pdf

2. Sánchez Romero S. Prevalencia de síndrome de Burnout en profesionales de enfermería de las Unidades de Cuidados Críticos y Urgencias del Hospital médico Quirúrgico de Jáen. Revista Enfermería del Trabajo. 2014; 4: 115-125. https://dialnet.unirioja.es/servlet/articulo?codigo $=5213004$

3. Alba Martin R. Burnout en enfermería: prevalencia y factores relacionados en el medio hospitalario. Revista Científica de la Sociedad Española de Enfermería Neurológica. 2015;41(1):9-14.

4. Soto Cámara R, Santamaría Cuesta MI. Prevalencia del burnout en la enfermería de atención primaria. Enfermería Clínica (España).

2005; 15(03):123-30. https://dialnet.unirioja.es/servlet/articulo?codigo=1182507 
5. Gómez de Regil L., Estrella-Castillo D. Burnout: una revisión breve. Revista Ciencia y Humanismo en la Salud. 2015, Vol. 2, No 3, pp. 116-122.

6. Salillas R. Síndrome de Burnout en profesionales de enfermería en el ámbito hospitalario: un estudio descriptivo. Revista Enfermería del Trabajo. 2017. 7; 3: 65-69. https://dialnet.unirioja.es/servlet/articulo?codigo=6112224

7. Cruz Robazzi MLdC, Chaves Mauro MY, Barcellos Dalri RdCdM, Almeida da Silva $L$, de Oliveira Secco IA, Jorge Pedrão L. Exceso de trabajo y agravios mentales a los trabajadores de la salud. Revista Cubana de Enfermería. 2010;26:52-64. http://scielo.sld.cu/scielo.php?script=sci arttext\&pid=S0864-03192010000100009

8. Canales-Vergara M, Valenzuela-Suazo S, Paravic-Klijn T. Condiciones de trabajo de los profesionales de enfermería en Chile. Enfermería Universitaria. 2016;13(3):178-86.

9. McGahan M, Kucharski G, Coyer F, Paper WABNR. Nurse staffing levels and the incidence of mortality and morbidity in the adult intensive care unit: a literature review. Australian Critical Care. 2012;25(2):64-77. https://www.ncbi.nlm.nih.gov/pubmed/22515951

10. Kane RL, Shamliyan TA, Mueller C, Duval S, Wilt TJ. The association of registered nurse staffing levels and patient outcomes: systematic review and metaanalysis. Medical care. 2007;45(12):1195-204. https://www.ncbi.nlm.nih.gov/pubmed/18007170

11. Bargas EB, Monteiro MI. Factors Related To Absenteeism Due To Sickness In Nursing Workers [fatores Relacionados Ao Absenteísmo Por Doença Entre Trabalhadores De Enfermagem]. Acta Paulista de Enfermagem. 2014. http://www.scielo.br/scielo.php?script=sci arttext\&pid=S0103-21002014000600533

12. Balseiro Almario L. El síndrome de Burnout : como factor de riesgo laboral en el personal de enfermería. México: Trillas; $2010 . \quad$ http://revistaenfermeria.unam.mx/ojs/index.php/enfermeriauniversitaria/article/view/458

13. Puialto Durán MJ, Antolín Rodríguez R, Moure Fernández L. Prevalencia del síndrome del quemad@ y estudio de factores relacionados en I@s enfermer@s del chuvi (Complexo Hospitalario Universitario de Vigo). Enfermería global: Revista electrónica semestral de enfermería. 2006;5(1). https://digitum.um.es/digitum/bitstream/10201/24138/1/Prevalencia\%20del\%20Sindro me\%20del\%20Quemad\%20y\%20estudio\%20de\%20factores\%20relacionados\%20en \%20\%20l\%20s\%20enfermers\%20del\%20CHUVI..pdf

14. Olivares-Faundez VE, Mena-Miranda L, Jelvez-Wilke C, Macia-Sepulveda F. Validez factorial del Maslach Burnout Inventory human services (MBI-HSS) en profesionales Chilenos. Univ Psychol Universitas Psychologica. 2014;13(1):145-60. http://www.scielo.org.co/pdf/rups/v13n1/v13n1a13.pdf

15. Hernández Vargas $\mathrm{Cl}$, Llorens Gumbau S, Rodríguez Sánchez AM. Burnout en personal sanitario: validación de la escala MBlen México. Forum de recerca. 2011(16):1-10. https://dialnet.unirioja.es/servlet/articulo?codigo=4833552

16. Comisión Nacional de Bioetica. Actualizacion de la Declaracion de Helsinki y su impacto en la investigación en seres humanos. Universidad Autonoma del Estado de Hidalgo. 2018.

https://www.uaeh.edu.mx/investigacion/productos/4821/libro principios de etica.pdf 17. Castillo-Barrera B, Solano-Pérez CT, Solano-Solano G, Pérez LED, BustoVillarreal JM, Barrera-Gálvez R. Personal de enfermería con cansancio emocional: Una muestra del hospital general de Tulancingo, México. Educación y Salud Boletín Científico de Ciencias de la Salud del ICSA. 2016;4(8). https://repository.uaeh.edu.mx/revistas/index.php/ICSA/article/view/279 
18. Aldrete Rodríguez MG, Contreras Estrada MI, González Baltazar R, Navarro Meza C, Pérez Aldrete J. Factores psicosociales y síndrome de burnout en personal de enfermería de una unidad de tercer nivel de atención a la salud. cyt Ciencia \& trabajo.

2015;17(52):32-6. https://scielo.conicyt.cl/scielo.php?script=sci arttext\&pid=S0718-24492015000100007 19. Contreras-Palacios SdC, Álvarez-Gárcia MI, Priego-Álvarez HR, MoralesGarcía MH, Córdova-Hernández JA. Síndrome de Burnout, funcionalidad familiar y factores relacionados en personal médico y de enfermería de un hospital de Villahermosa, Tabasco, México. Horizonte sanitario. 2013;12(2):45-57. http://revistas.ujat.mx/index.php/horizonte/article/view/238

20. Zavala-González MA, Eduardo P-AS, Oscar J-M, Leonor L-MR, Guadalupe P$\mathrm{RL}$, Beatriz P-AM. Síndrome de Burnout en personal médico y de enfermería de una unidad médica familiar en Tabasco, México. Revista Médica de la Universidad Veracruzana.

https://www.medigraphic.com/cgibin/new/resumen.cgi?IDARTICULO=35754

21. Grisales Romero $H$, Muñoz Y, Osorio Vélez DM, Robles E. Síndrome de Burnout en el personal de enfermería de un hospital de referencia Ibagué, Colombia, 2014. Enfermería global: Revista electrónica semestral de enfermería. 2016;15(1):244-71. https://revistas.um.es/eglobal/article/view/212851

22. Maruy-Saito A, Verne-Martin E, Vásquez-Manrique JF. Frecuencia del síndrome de burnout y niveles de sus dimensiones en el personal de salud del servicio de emergencia de pediatría del Hospital Nacional Cayetano Heredia en el año 2014: Lima, Perú. rnp Revista de Neuro-Psiquiatría. 2014;77(3):168-74 http://www.upch.edu.pe/vrinve/dugic/revistas/index.php/RNP/article/view/2031

23. Sánchez Alonso P, Sierra Ortega VM. Síndrome de Burnout en el personal de enfermería en UVI. Enfermería global: Revista electrónica semestral de enfermería. 2014;13(1):252-66. http://scielo.isciii.es/scielo.php?script=sci arttext\&pid=S169561412014000100012

24. Arias Gallegos WL, Muñoz del Carpio Toia A. Síndrome de burnout en personal de enfermería de Arequipa. Revista Cubana de Salud Pública. 2016;42(4):0-20. http://scielo.sld.cu/scielo.php?script=sci arttext\&pid=S0864-34662016000400007

25. Fuentelsaz-Gallego C, Moreno-Casbas T, Gómez-García T, González-María E, Consorcio RNC-E. Entorno laboral, satisfacción y burnout de las enfermeras de unidades de cuidados críticos y unidades de hospitalización. Proyecto RN4CASTEspaña. Enfermería intensiva / Sociedad Española de Enfermería Intensiva y Unidades Coronarias. 2013;24(3).

26. Álvarez Verdugo LP, Prieto Bocanegra BM. Prevalencia de desgaste profesional en personal de enfermería de un hospital de tercer nivel de Boyacá, Colombia. Enfermería global: Revista electrónica semestral de enfermería. 2013;12(1):73-88. $\quad$ http://scielo.isciii.es/scielo.php?script=sci abstract\&pid=S169561412013000100004

27. Miranda Lara VR, Monzalvo Herrera G, Hernández Caballero B, Ocampo Torres M. Prevalencia del síndrome de burnout en personal de enfermería de dos instituciones de salud. Revista Enfermería Instituto Mexicano Seguro Social. 2016;2:115-22. $\quad$ https://www.medigraphic.com/pdfs/enfermeriaimss/eim2016/eim162g.pdf

28. Garcia-Campayo J, Puebla-Guedea M, Herrera-Mercadal P, Dauden E. Burnout Syndrome and Demotivation Among Health Care Personnel. Managing Stressful Situations: The Importance of Teamwork. Actas Dermosifiliogr.2016;107(5):400-6.27 
ISSN 1695-6141

๑) COPYRIGHT Servicio de Publicaciones - Universidad de Murcia 